\title{
$\beta$-elemene inhibits stemness, promotes differentiation and impairs chemoresistance to temozolomide in glioblastoma stem-like cells
}

\author{
TING-ZHUN ZHU ${ }^{1}$, XIAO-MING LI ${ }^{1}$, LI-HAN LUO $^{2}$, ZHEN-QUAN SONG $^{1}$, \\ $\mathrm{XU} \mathrm{GAO}^{1}$, ZHI-QING LI ${ }^{1}$, JING-YUAN SU ${ }^{1}$ and GUO-BIAO LIANG ${ }^{1}$ \\ ${ }^{1}$ Department of Neurosurgery, General Hospital of Shenyang Military Area Command, Shenyang 110840; \\ ${ }^{2}$ Health Care Centre, Shenyang Entry-Exit Inspection and Quarantine Bureau, Shenyang 110016, P.R. China
}

Received January 27, 2014; Accepted March 4, 2014

DOI: 10.3892/ijo.2014.2448

\begin{abstract}
Accumulating evidence indicates that glioblastoma stem-like cells (GSCs) are key factors in tumour development, recurrence and chemoresistance. The impairment of stemness and the enhancement of differentiation contributes to the weakening of radiation and chemotherapy resistance of GSCs. We previously found that $\beta$-elemene was an effective anti-glioblastoma agent and chemosensitizer. In this study, we examined the distribution of $\mathrm{CD}_{13} 3^{+}$cells in human glioblastoma tissues by immunohistochemistry. Following treatment with $\beta$-elemene, the formation of GSC spheres was investigated by manual counting, the proliferation of GSCs was measured with a Cell Counting Kit-8 (CCK-8) assay, and the dispersion of GSC spheres was observed with an inverted microscope. GSC spheres were treated with $\beta$-elemene, and the expression levels of CD133, ATP-binding cassette subfamily G member 2 (ABCG2) and glial fibrillary acidic protein (GFAP) were examined by western blotting. After treatment with $\beta$-elemene, the volumes and weights of GSC xenografts were measured, and the expression of CD133, ABCG2 and GFAP was evaluated through immunohistochemistry analysis. After treatment with $\beta$-elemene and temozolomide (TMZ), GSC viability was examined by the CCK- 8 assay, and the volumes and weights of xenografts were measured. We found that $\mathrm{CD} 133^{+}$cells were assembled in some vascular walls and also sparsely distributed in other parts of glioblastoma tissues. $\beta$-elemene decreased the formation of GSC spheres, dispersed GSC spheres and inhibited the proliferation of GSCs in vitro and in vivo. In the GSC spheres and xenografts treated with $\beta$-elemene, the expression
\end{abstract}

Correspondence to: Professor Guo-Biao Liang, Department of Neurosurgery, General Hospital of Shenyang Military Area Command, No. 83, Wenhua Road, Shenhe District, Shenyang 110840, P.R. China

E-mail: syjqzyyliangguobiao@yahoo.com

Key words: $\beta$-elemene, glioblastoma stem-like cell, stemness, differentiation, chemoresistance of CD133 and ABCG2 was significantly downregulated, and the expression of GFAP increased. Furthermore, the sensitivity of GSCs to TMZ was enhanced in vitro and in vivo. These results suggest that $\beta$-elemene impaired the stemness of GSC spheres, promoted their differentiation and sensitized GSCs to TMZ. $\beta$-elemene will hopefully become a valuable agent to enhance the effects of radiotherapy and chemotherapy.

\section{Introduction}

Glioblastoma is the most common and lethal type of primary brain tumours, accounting for approximately $52 \%$ of primary intracranial tumours. Glioblastoma is characterised by strong chemotherapy resistance and post-operative recurrence with a median survival of less than 12 months. Despite many advances in therapeutics over the past several years, the prognosis for patients with glioblastoma remains dismal (1-5).

In the past decade, accumulating evidence has indicated that glioblastoma stem-like cells (GSCs, also known as glioblastoma initiating cells) are at the root of tumour development, recurrence and drug resistance (6). GSCs are a small population $(1-10 \%)$ of cells with some neural stem cell (NSC) properties. GSCs have limitless self-renewal capacity and express some stem cell hallmarks, such as CD133, ATP-binding cassette subfamily G member 2 (ABCG2) and nestin. Additionally, GSCs are multipotent and can differentiate into neurons, astroglial cells and oligodendroglial cells to thus sculpt a resulting tumour that is composed of a mixture of different cell types. GSCs also have the ability to initiate and drive tumour progression in animal models $(7,8)$. These characteristics have been used to identify GSCs in many studies (9-11).

GSCs frequently show strong radioresistance and chemoresistance. It has been suggested that conventional post-operative chemotherapy and radiotherapy will kill most remaining cancer cells but will leave GSCs intact, resulting in the recurrence of glioblastoma. It has been reported that the radioresistance and chemoresistance of tumour stem cells (TSCs) is markedly decreased after their differentiation into normal tumour cells that display certain differentiation hallmarks $(12,13)$. Thus, finding effective ways to promote GSC differentiation will hopefully limit the radiotherapy 
and chemotherapy resistance of GSCs and notably enhance therapeutic efforts.

For use as an effective antitumour medicine, elemene is extracted from Curcuma wenyujin as an essential oil mixture of $\beta$-, $\gamma$ - and $\delta$-elemene (14). $\beta$-elemene (molecular formula $\mathrm{C}_{15} \mathrm{H}_{24}$, molecular weight 204.34), the major active anticancer component in the elemene mixture, has strong anti-proliferative activity and induces apoptosis in various tumours, such as glioma, breast carcinoma and leukaemia $(15-17)$. We previously found that $\beta$-elemene can inhibit the proliferation of glioblastoma cells and induce cell apoptosis in vitro and in vivo (17-21). In addition, $\beta$-elemene can promote the differentiation of Tca8113 human tongue squamous cancer cells and PLA801D human pulmonary giant cell carcinoma cells in vitro $(22,23)$. Therefore, it was essential to illustrate the effects of $\beta$-elemene on GSC proliferation, differentiation and chemoresistance.

In this study, we illustrated the effect of $\beta$-elemene on some stem cell phenotypes of GSCs. We found that CD133+ cells were not only assembled in some vascular walls but were also sparsely localized to other zones of glioblastoma tissues. $\beta$-elemene inhibited the proliferation of GSCs in vitro and in vivo, decreased the formation of GSC spheres, dispersed GSC spheres and even resulted in the fragmentation and death of some sphere cells. $\beta$-elemene significantly downregulated the expression levels of CD133 and ABCG2 and increased the expression of the astroglial cell marker glial fibrillary acidic protein (GFAP) in vitro and in vivo. Additionally, treatment with $\beta$-elemene sensitized GSCs to temozolomide (TMZ). These results suggested that $\beta$-elemene could impair the stemness of GSCs and promote their differentiation, which may make it a useful tool to enhance the effects of radiotherapy and chemotherapy.

\section{Materials and methods}

Reagents and antibodies. $\beta$-elemene (98\% purity) was obtained from Jingang Pharmaceutical Co. (Dalian, China). TMZ and 4',6-diamidino-2-phenylindole (DAPI) were obtained from Sigma-Aldrich Co., LLC. (St. Louis, MO, USA). The antibodies against CD133, ABCG2, GFAP, neuron specific enolase (NSE), and myelin basic protein (MBP), used in western blots and immunofluorescence assays, were purchased from Boster Co., Ltd. (Wuhan, China). Epidermal growth factor (EGF), basic fibroblast growth factor (bFGF), leukaemia inhibitory factor (LIF), N2, Accutase Cell Dissociation Reagent and Alexa Fluor 594 goat anti-rabbit IgG were from Invitrogen Corp. (Carlsbad, CA, USA). The antibody against GAPDH was from Santa Cruz Biotechnology, Inc. (Santa Cruz, CA, USA). Anti-CD133 and IgG1 isotype control antibodies used in flow cytometry analysis were purchased from Miltenyi Biotec (Bergish Gladbach, Germany). The Cell Counting Kit-8 (CCK-8) was obtained from Dojindo Molecular Technologies, Inc. (Japan). Nude mice were provided by the Experimental Animal Center of The Academy of Military Medical Sciences. Primary glioblastoma cells were maintained in Dulbecco's modified Eagle's medium (DMEM)/F12 (Hyclone, UT, USA) supplemented with $10 \%$ foetal calf serum, $50 \mathrm{IU} / \mathrm{ml}$ penicillin and $50 \mathrm{mg} / \mathrm{ml}$ streptomycin and grown at $37^{\circ} \mathrm{C}$ in a humidified atmosphere with $5 \% \mathrm{CO}_{2}$.
Tumour specimens and primary cell cultures. Tumour tissues originated from 20 patients with glioblastoma (10 men and 10 women, $42 \pm 11.7$ years, World Health Organization/WHO grade III-IV) undergoing surgical resection in the Department of Neurosurgery of the General Hospital of Shenyang Military. The cells of two of these patients (case 1, WHO grade III; and case 2, WHO grade IV) were cultured as primary glioblastoma cells. The study was approved by the Ethics Committee of the General Hospital of Shenyang Military and abided by the Declaration of Helsinki, with informed consent obtained from all study participants. Tumour samples were stored in sterile serum-free DMEM/F12 and processed within $0.5 \mathrm{~h}$ after resection. Tissues were cut into 1-mm ${ }^{2}$ pieces, washed with PBS and digested with $0.25 \%$ trypsin at $37^{\circ} \mathrm{C}$ for $15 \mathrm{~min}$. Cells were cultured in serumcontaining DMEM/F12 after passage through a $70-\mu \mathrm{m}$ strainer (BD Pharmingen, San Diego, CA, USA). Primary glioblastoma cells were resuspended in NSC medium (NSCM) containing DMEM/F12 medium, $20 \mathrm{ng} / \mathrm{ml}$ EGF, $20 \mathrm{ng} / \mathrm{ml}$ bFGF, $10 \mathrm{ng} / \mathrm{ml}$ LIF and N2 (1:100) for culturing GSCs.

Immunohistochemistry. Paraffin sections of glioblastoma tissues were obtained and deparaffinized. Antigen retrieval was performed with citrate buffer, $\mathrm{pH} 6.0$ (Invitrogen). Non-specific sites were blocked by incubating sections with 5\% BSA in a humidified chamber for $1 \mathrm{~h}$ at room temperature. The samples were incubated with $0.3 \% \mathrm{H}_{2} \mathrm{O}_{2}$ for 15 min to block endogenous peroxidase activity and then labelled with different primary antibodies at proper concentrations (1:100 for CD133, ABCG2 and GFAP) for $1 \mathrm{~h}$ at room temperature. In subsequent steps, we used the Vectastain ABC kit (Vector Laboratories, Burlingame, CA, USA) and DAB as a chromogen (Changdao Biotech, China). The nuclear counterstaining of sections was performed with hematoxylin. In every tissue, additional staining without primary antibody was performed in parallel as a negative control. The integrated optical density (IOD) was used to semi-quantitatively estimate the expression of CD133, ABCG2 and GFAP in Image-Pro Plus 6.0.

Determining the CD133 expression by flow cytometry analysis. Cells were digested into single cells with Accutase Cell Dissociation Reagent and labelled with anti-CD133 and control IgG isotype monoclonal antibodies (Miltenyi Biotec, San Diego, CA, USA). Flow cytometry analysis was conducted on a FACSCalibur flow cytometer (Becton-Dickinson, San Jose, CA, USA). Data acquisition and analysis were performed with CellQuest software (Becton-Dickinson).

GSC culture and passage. Primary glioblastoma cells were cultured in NSCM and formed cell spheres after 3 days. After forming spheres of at least 100 cells (culturing for approximately 5 days), cell spheres were digested to single cell suspension with Accutase Cell Dissociation Reagent and subcultured with NSCM in ultra-low attachment culture flasks (Corning, Inc., NY, USA).

Immunofluorescence and DAPI staining. In each well of 24-well plates, $2 \times 10^{5}$ dissociated cells or 20 cell spheres were cultured on poly-L-lysine-coated cover slips for various times. Cells on cover slips were washed with PBS, fixed with methanol and then treated with $0.1 \%$ Triton X-100. After blocking 

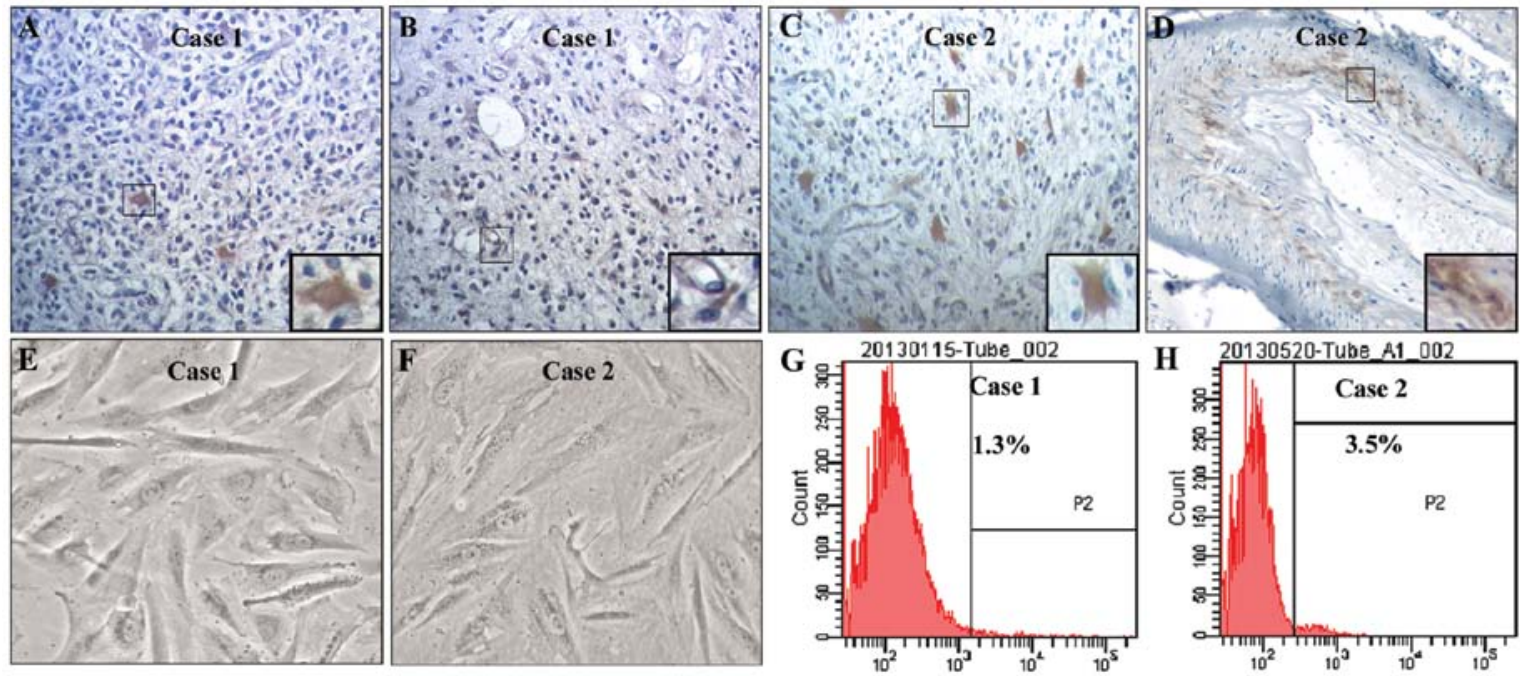

Figure 1. The distribution of $\mathrm{CD} 133^{+}$cells in human glioblastoma tissues and the proportion of $\mathrm{CD}_{133^{+}}$cells in primary glioblastoma cells. (A-D) CD133 ${ }^{+}$cells were not only assembled in some vascular walls but were also sparsely localized in other zones of glioblastoma tissues (x100). An individual tumour cell (inset black squares) was magnified to facilitate the interpretation of the staining pattern. (E and F) Primary glioblastoma cells were obtained from case 1 and case 2 (x200). ( $\mathrm{G}$ and $\mathrm{H})$ The proportions of $\mathrm{CD}_{133^{+}}$cells in primary glioblastoma cells were $1.3 \%$ for case 1 and $3.5 \%$ for case 2 .

with 5\% BSA, cells were incubated with proper dilutions of anti-CD133, GFAP, MBP or NSE antibodies at $4^{\circ} \mathrm{C}$ overnight. The primary antibodies were detected with Alexa Fluor 594 goat anti-rabbit IgG (dilution 1:500) for $40 \mathrm{~min}$ at $37^{\circ} \mathrm{C}$ in the dark. Cell nuclei were stained with DAPI, and immunofluorescent images were examined under a fluorescent microscope (Leica Microsystems, Germany).

Cell proliferation assay. Cell viability was evaluated with the CCK-8 assay after cells in exponential growth were cultured in a 96-well plate. Trypan blue staining confirmed $>80 \%$ viability, and cells were treated according to the study design. Then, $10 \mu 1$ of CCK- 8 was added to each well, and the mixture was incubated for $4 \mathrm{~h}$ at $37^{\circ} \mathrm{C}$. The optical density (OD) of each well was measured at $450 \mathrm{~nm}$ using a spectrophotometric microplate reader (BioTek Instruments, Inc., Winooski, VT, USA). Five replicate wells were used for each cell sample.

Western blotting. The cells were lysed with RIPA buffer $[50 \mathrm{mM}$ Tris- $\mathrm{HCl}$ (pH 7.4), 1.0\% NP-40, 0.25\% Na-deoxycholate, $1 \mathrm{mM}$ EDTA, $150 \mathrm{mM} \mathrm{NaCl}, 1 \mathrm{mM}$ aprotinin, $1 \mathrm{mg} / \mathrm{ml}$ PMSF, $1 \mu \mathrm{g} / \mathrm{ml}$ pepstatin and $1 \mu \mathrm{g} / \mathrm{ml}$ leupeptin]. The concentrations of total protein in the cellular extracts were measured using the BCA assay kit from Keygen Biotech, Co., Ltd. (Nanjing, China). After separation in 10-12\% sodium dodecyl sulphate polyacrylamide (SDS-PAGE) gels, the proteins were transferred to nitrocellulose filter membranes (Bio-Rad, Hercules, CA, USA). The membranes were blocked with $5 \%$ BSA in Tris-buffered saline with Tween-20 at $4^{\circ} \mathrm{C}$ overnight and probed with various primary antibodies at $4{ }^{\circ} \mathrm{C}$ overnight, followed by incubation with horseradish peroxidase-conjugated secondary antibodies at $37^{\circ} \mathrm{C}$ for $3 \mathrm{~h}$. The membranes were exposed to an ECL system (Amersham, Sweden), and fluorescence was detected by exposing the membrane to X-ray film. The results were scanned with the Image Quant 5.2 software (Amersham).
Transplantation of GSCs into nude mice and treatment of animals. GSC spheres were digested into single cells, and 1000 cells/animal (suspending in $0.1 \mathrm{ml}$ DMEM/F12) were subcutaneously injected into the right shoulder region of 4-weekold female nude mice. The use of animals was approved by the Institutional Animal Care and Use Committee. Beginning with the 7th day after transplantation, various drugs were intraperitoneally injected. The volumes of tumours were measured every 3 days and calculated according to the formula: $V=1 / 2 \times$ largest diameter $\mathrm{x}$ smallest diameter ${ }^{2}(21)$. Tumours were weighed on the 21st day after transplantation.

Statistical analysis. The values are reported as the means \pm standard deviation (SD) of at least three independent experiments. Statistical analysis was performed using Student's t-test. Statistical significance was accepted at the level of $\mathrm{P}<0.05$ between different groups, and $\mathrm{P}<0.01$ was considered highly significant. Statistical analyses were performed with SPSS software (SPSS, Inc., Chicago, IL, USA).

\section{Results}

The expression of CD133 in human glioblastoma tissues and the proportion of $\mathrm{CDI}_{3} 3^{+}$cells in primary glioblastoma cells. To investigate the distribution of $\mathrm{CD} 133^{+}$cells in human glioblastoma tissues, immunohistochemistry was performed with an anti-CD133 antibody. To examine the proportion of CD133+ primary glioblastoma cells, we cultured these cells from fresh glioblastoma tissues and performed flow cytometry using an anti-CD133 antibody. We found that $\mathrm{CD} 133^{+}$cells were not only assembled in some vascular walls but were also sparsely localized in other zones of glioblastoma tissues (Fig. 1A-D). Primary glioblastoma cells obtained from two glioblastoma samples (case 1 and case 2) had obvious cellular atypia and pathological nuclear fission (Fig. 1E and F). The proportions of $\mathrm{CD} 133^{+}$cells were $1.3 \%$ in case 1 (Fig. 1G) and $3.5 \%$ in case 2 (Fig. 1H). 

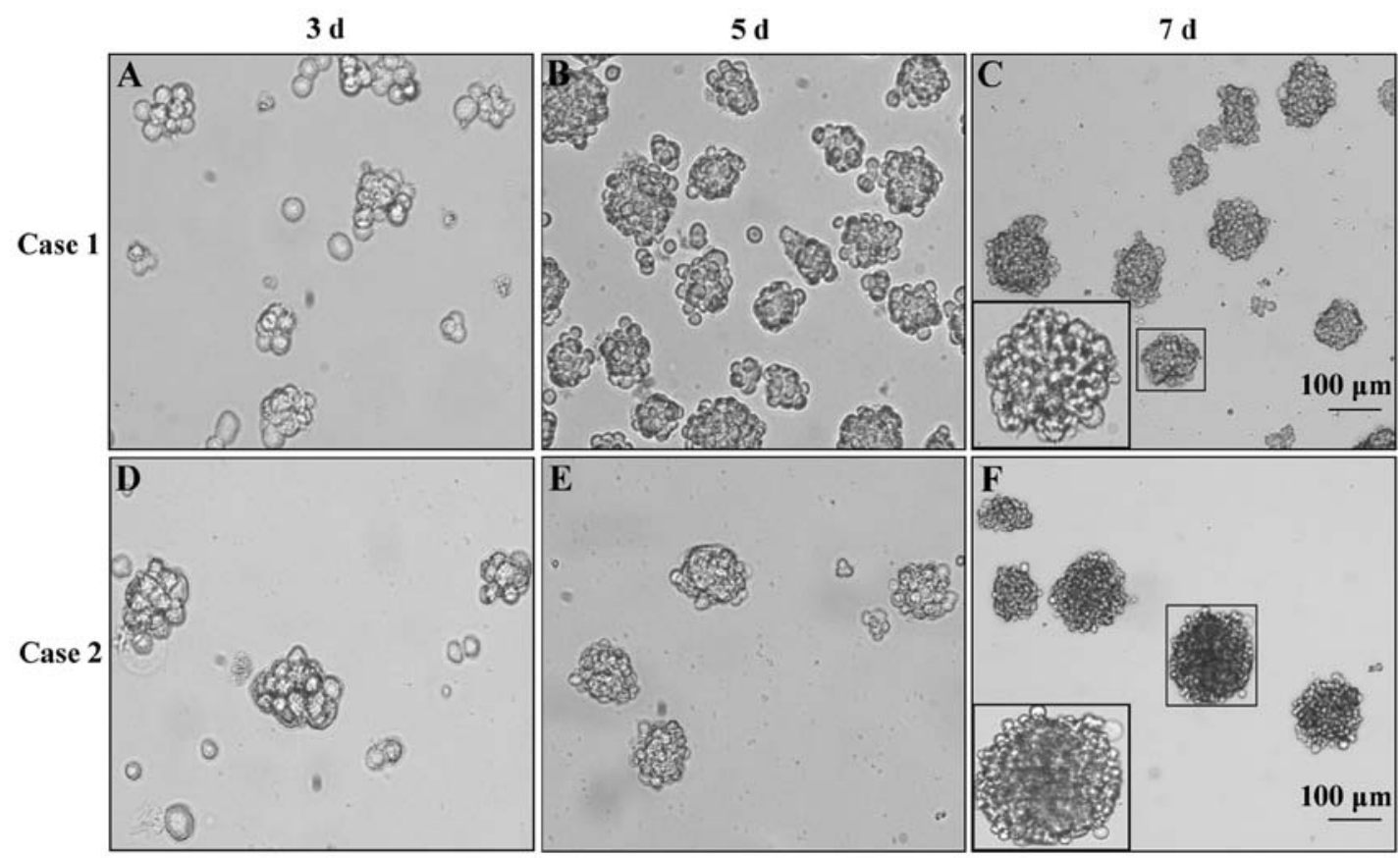

Figure 2. The formation of second-generation GSC spheres. (A) The second-generation GSC spheres of case 1 formed on the 3rd day and contained approximately 20 cells each. (B) Spheres of case 1 gradually expanded to 100 cells with a dense structure and high refractivity on the 5th day. (C) Spheres of case 1 continued to grow on the 7th day (the magnified sphere in black square). (D) The second-generation GSC spheres of case 2 formed on the 3rd day and contained approximately 30 cells each. (E) Spheres of case 2 gradually expanded to 100 cells with a dense structure and high refractivity on the 5th day. (F) Spheres of case 2 continued to grow on the 7th day (the magnified sphere in black square). Original magnifications x100. Scale bar, $100 \mu \mathrm{m}$.

The formation of second-generation GSC spheres. To obtain GSC spheres, we cultured primary glioblastoma cells in NSCM and observed cell sphere formation with an inverted microscope. The results showed that primary glioblastoma cells derived from case 1 and case 2 could form GSC spheres in NSCM. The second-generation GSC spheres formed on the 3rd day after passage and then grew gradually with a denser structure and higher refractivity (Fig. 2).

GSC spheres strongly express the NSC marker CD133 and show multipotent differentiation capacity. To test the expression of CD133 and the multipotent differentiation capacity in the second-generation GSC spheres, we cultured GSC spheres in DMEM/F12 with 10\% foetal calf serum and examined CD133, GFAP (astrocyte marker), MBP (oligodendrocyte marker) and NSE (neuron marker) expression by immunofluorescence assays 0,48 and $96 \mathrm{~h}$ after plating. We found that the secondgeneration GSC spheres strongly expressed CD133 with little expression of GFAP, MBP and NSE (Fig. 3, 0 h). During $48 \mathrm{~h}$ of adherent growth conditions in serum-containing DMEM/F12, GSC spheres gradually spread out. The expression of CD133 decreased, whereas the expression of GFAP, MBP and NSE increased with time. After 96 h, GFAP, MBP and NSE showed strong expression (Fig. 3). These results suggest that the GSC spheres we obtained possessed stem cell phenotypes and multipotent differentiation potential.

$\beta$-elemene inhibits the formation of GSC spheres, decreases the proliferation of GSCs and disperses cell spheres. To evaluate the effect of $\beta$-elemene on GSC sphere formation, cell spheres were digested into single cells. The cells were then plated at a density of 100 cells/well in 96-well plates and simultaneously treated with $100 \mu \mathrm{g} / \mathrm{ml} \beta$-elemene for $72 \mathrm{~h}$ (untreated cells as control group). Finally, the number of cell spheres (containing at least 10 cells/sphere) was counted. To examine the effect of $\beta$-elemene on proliferation, 1000 GSCs and 10000 primary glioblastoma cells per well were independently plated in 96 -well plates. These cells were then treated with 0 (control group), 50, 100, 150 or $200 \mu \mathrm{g} / \mathrm{ml} \beta$-elemene for $24 \mathrm{~h}$, and the CCK-8 assay was used to examine cell viability. To test the ability of $\beta$-elemene to dissociate GSC spheres, we added $200 \mu \mathrm{g} / \mathrm{ml} \beta$-elemene to cell spheres for $12 \mathrm{~h}$ and observed sphere dispersion with an inverted microscope. The results showed that the number of GSC spheres that formed in the $\beta$-elemene group was less than in the control groups in both cases (Fig. 4A, P<0.01). The proliferation of both GSCs and primary glioblastoma cells (GC) (Fig. 4B) decreased dosedependently in the presence of $\beta$-elemene. The degree of this viability loss was less for GSCs than for primary glioblastoma cells (Fig. 4B), which suggested that GSCs possessed stronger resistance to $\beta$-elemene than glioblastoma cells. $\beta$-elemene can disperse GSC spheres, and this can result in the fragmentation and death of some cells (Fig. 4C-F).

$\beta$-elemene decreases the expression of stem cell markers $C D 133$ and $A B C G 2$ and increases the expression of the differentiation marker GFAP in GSC spheres. To investigate the effect of $\beta$-elemene on the expression of GSC markers CD133 and ABCG2 and differentiation marker GFAP, GSC spheres were treated with 100 and $200 \mu \mathrm{g} / \mathrm{ml} \beta$-elemene for $24 \mathrm{~h}$, and western blot analysis was performed using specific antibodies. The results were semi-quantitatively estimated using Gel-Pro 


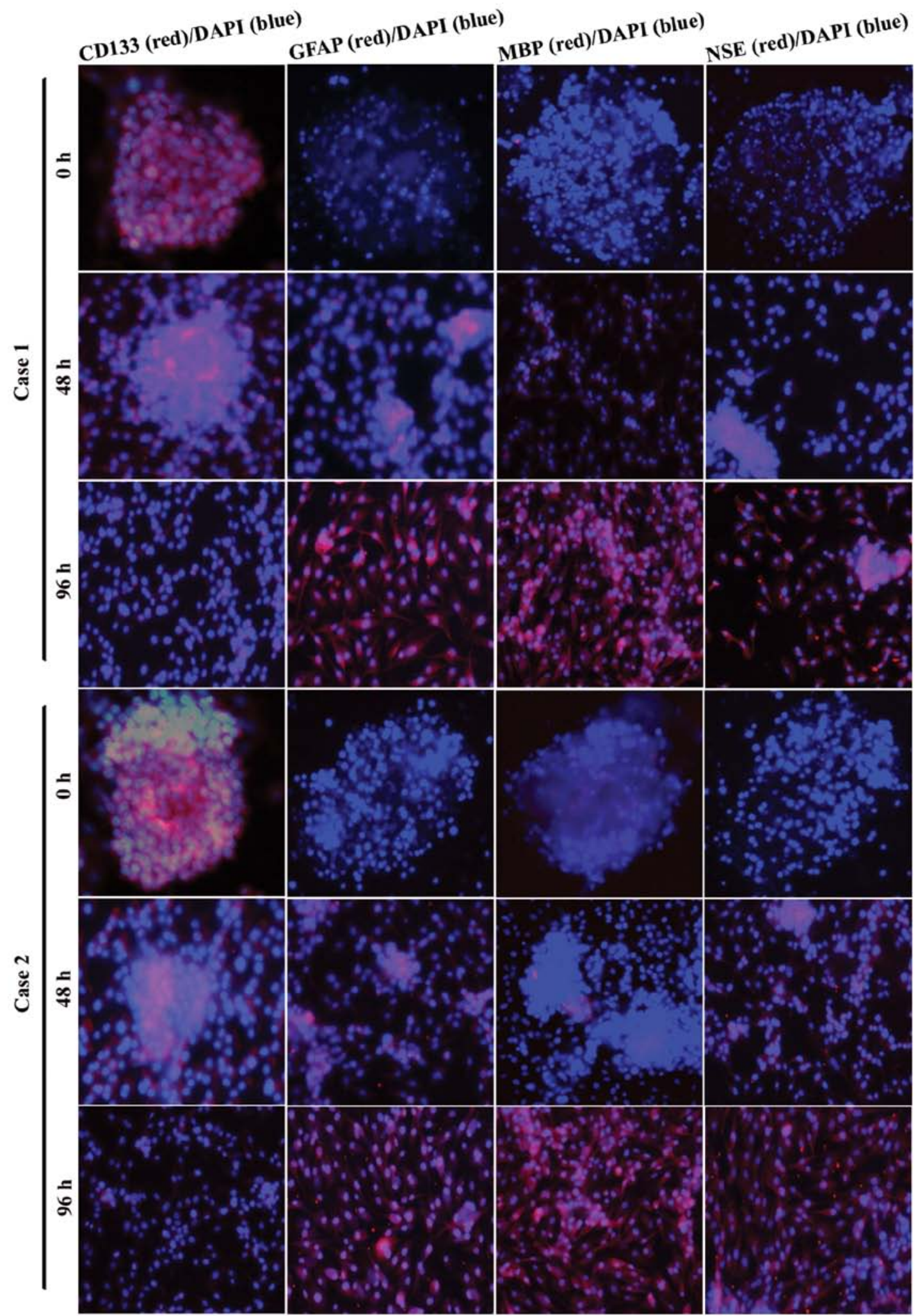

Figure 3. GSC spheres strongly expressed CD133 and showed multipotent differentiation potential. CD133, GFAP, MBP and NSE were examined in GSC spheres by immunofluorescence analysis 0,48 and $96 \mathrm{~h}$ after plating. The results showed that CD133 was strongly expressed in the second-generation GSC spheres with little GFAP, MBP and NSE $(0 \mathrm{~h})$. GSC spheres adherently grew and gradually spread to the surroundings at $48 \mathrm{~h}$. The expression of CD133 weakened, whereas the expression of GFAP, MBP and NSE increased with time. GFAP, MBP and NSE showed strong expression at $96 \mathrm{~h}$. This result suggested that the GSC spheres we obtained possessed stem cell phenotypes and multipotent differentiation potential. Original magnifications x100.

analyzer 4.0 software and illustrated graphically. We found that the expression levels of CD133 and ABCG2 were significantly downregulated, while the expression of GFAP was increased by $\beta$-elemene in a dose-dependent manner (Fig. 5). These results suggested that $\beta$-elemene impairs the stemness of GSC spheres and promotes their differentiation. 

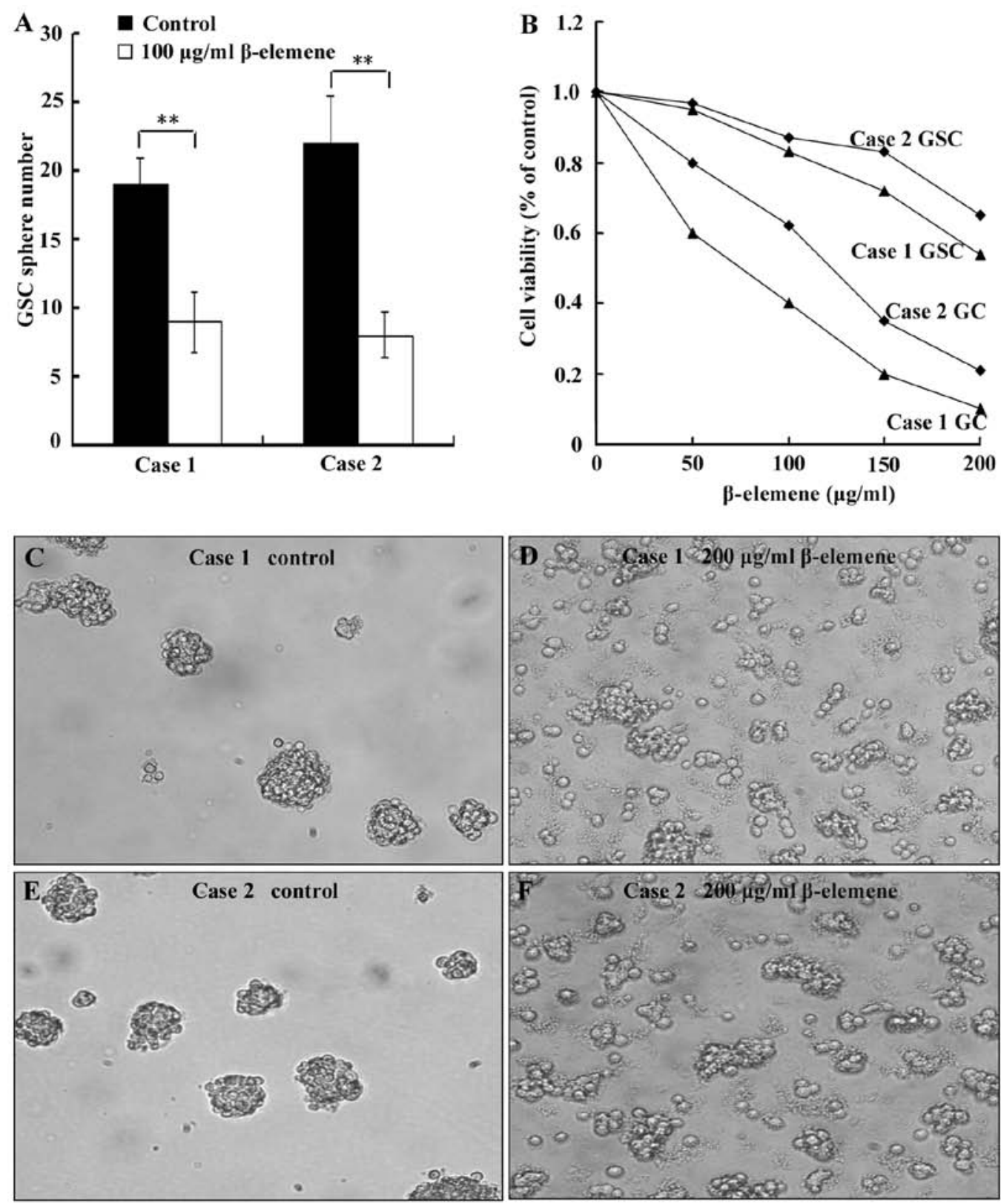

Figure 4. $\beta$-elemene inhibits the formation of GSC spheres, decreases the viability of GSCs and disperses cell spheres. (A) The number of GSC spheres that formed in the $\beta$-elemene group was less than in the control groups in both cases. (B) The proliferation of both GSCs and primary glioblastoma cells (GC) decreased dose-dependently in the presence of $\beta$-elemene. The degree of viability loss was less in GSCs than in primary glioblastoma cells, which suggested that GSCs are more resistant to $\beta$-elemene than glioblastoma cells. (C-F) GSC spheres were dispersed, and partial cells fragmented and died after $\beta$-elemene treatment in both cases. Values are shown as the means $\pm \mathrm{SD}\left({ }^{*} \mathrm{P}<0.05,{ }^{* *} \mathrm{P}<0.01\right)$.

$\beta$-elemene suppresses the development of tumours in nude mice transplanted with GSCs. To evaluate the effect of $\beta$-elemene on GSC tumours in vivo, we subcutaneously injected GSCs into the flank of nude mice and then intraperitoneally injected $\mathrm{NaCl}$ or $50 \mathrm{mg} / \mathrm{kg} \beta$-elemene for 1 week. The tumour volumes were measured every 3 days. Points are the means \pm SD of tumour volume in each group $(n=6)$. The results showed that a significant inhibition of tumour volume was observed in the $\beta$-elemene-treated group compared with the control group from the 9th day after transplantation (the 3rd day after intraperitoneal injection) to the 21st day, as shown in Fig. 6A and B. On the 21st day, tumours were dissected and measured. The tumour weights of the $\beta$-elemene group were significantly lower than those of the control group, as shown in Fig. 6C and D. These results suggested that the development of tumours was suppressed by $\beta$-elemene in GSC-bearing nude mice.

$\beta$-elemene downregulates the expression of $C D 133$ and $A B C G 2$ and upregulates the expression of GFAP in GSC-transplanted nude mice. To evaluate the effect of $\beta$-elemene on the expression of stem cell markers and differentiation markers in vivo, immunohistochemistry was performed using antibodies against $\mathrm{CD} 133, \mathrm{ABCG} 2$ and GFAP on the aforementioned tumour tissues of GSC-transplanted nude mice in $\beta$-elemene and $\mathrm{NaCl}$ groups (Fig. 7A). Image-Pro Plus 6.0 software was applied to calculate the IOD of each group (the same selected 
A

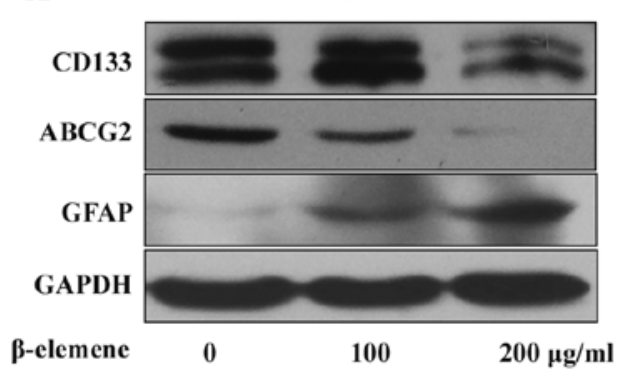

B

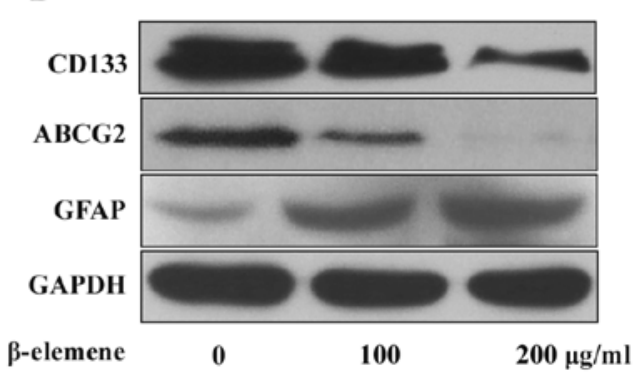

C

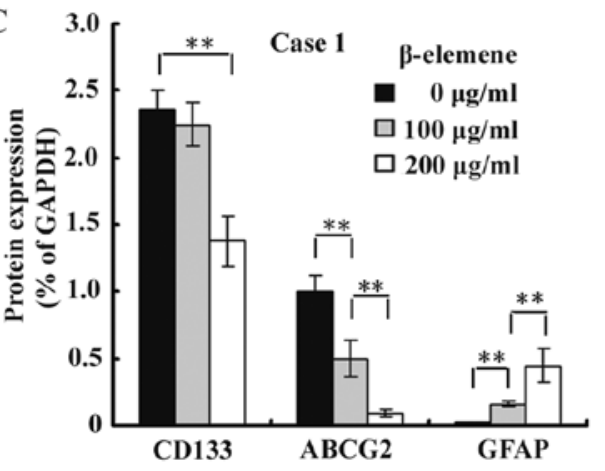

D

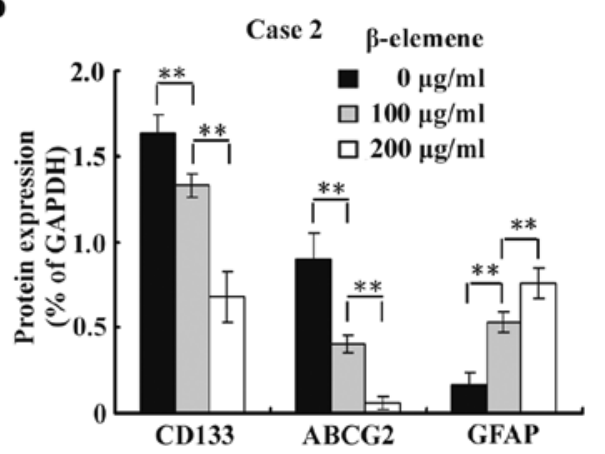

Figure 5. The expression of CD133 and ABCG2 is decreased, and the expression of GFAP is increased by $\beta$-elemene in GSC spheres. (A and B) $\beta$-elemene downregulated the expression of CD133 and ABCG2 and upregulated the expression of GFAP dose-dependently in case 1 and case 2. (C and D) The results of (A) and (B) were semi-quantitatively estimated using Gel-Pro Analyzer 4.0 software and illustrated in a polygram. The results are representative of three independent experiments, and values shown are the means $\pm \mathrm{SD}\left({ }^{*} \mathrm{P}<0.05,{ }^{* *} \mathrm{P}<0.01\right)$.

A

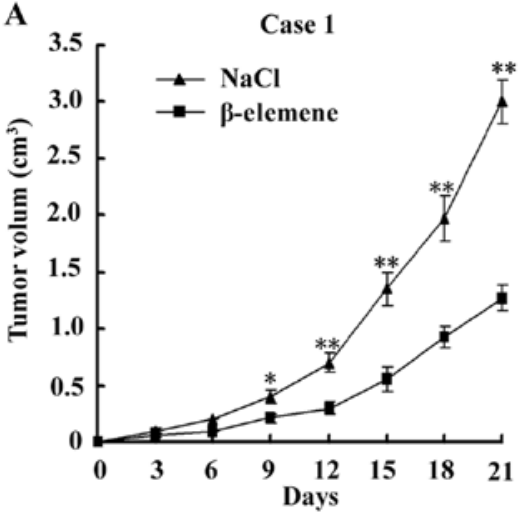

C

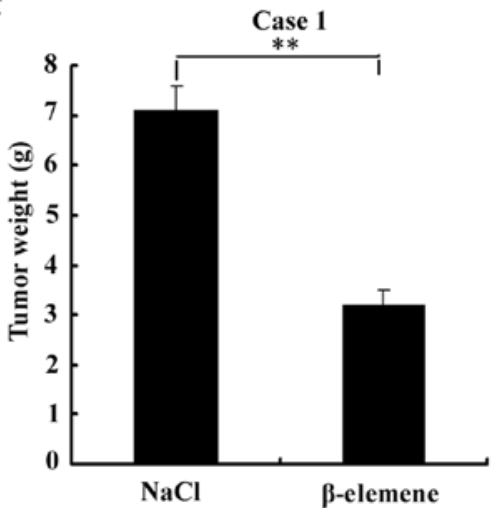

B

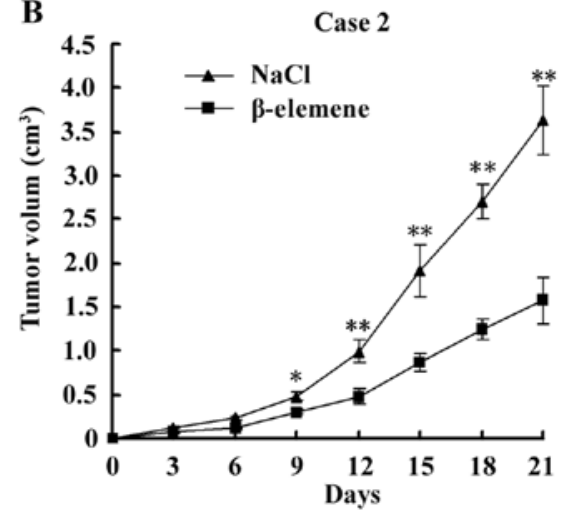

D

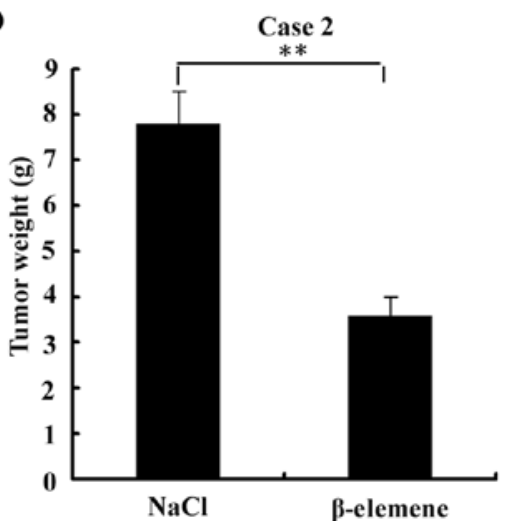

Figure 6. The development of tumours in nude mice transplanted with GSCs is suppressed by $\beta$-elemene. The nude mice transplanted with GSCs received an intraperitoneal injection of $\mathrm{NaCl}$ or $50 \mathrm{mg} / \mathrm{kg} \beta$-elemene for 1 week, and the tumour volumes were measured every 3 days. (A and B) In contrast to the control group, the remarkable suppression of tumour growth was observed for the whole period from the 9 th day after transplantation (the 3rd day after intraperitoneal injection) to the 21 st day in both cases 1 and 2. (C and D) The weights of tumours in the $\beta$-elemene group on the 21 st day after transplantation were significantly lower than those of the control group in both cases 1 and 2 . Values shown are the means $\pm \mathrm{SD}\left({ }^{*} \mathrm{P}<0.05,{ }^{* *} \mathrm{P}<0.01\right)$. 
A Case 1

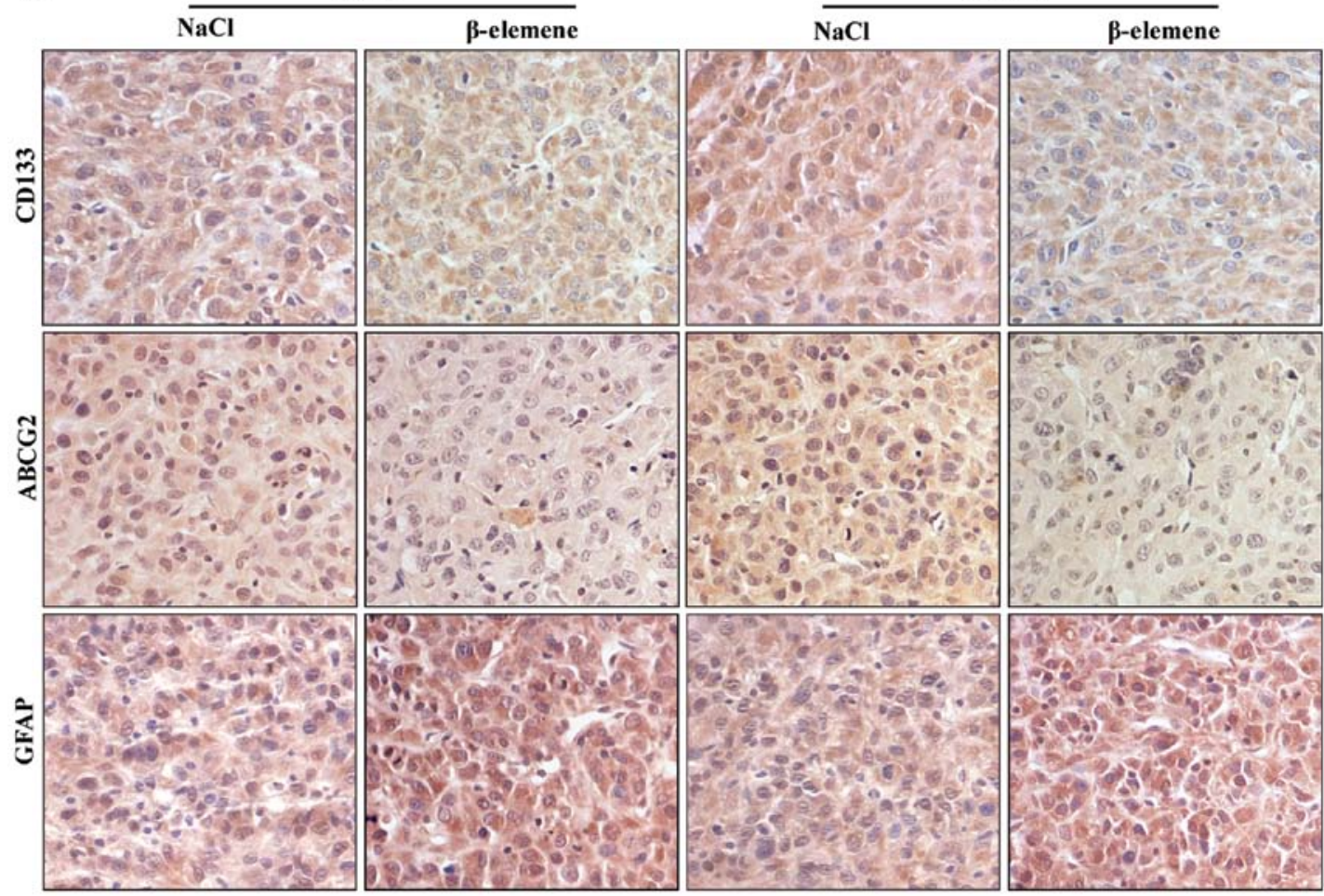

B

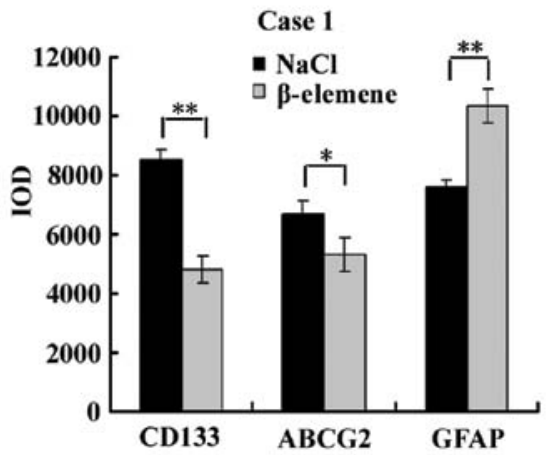

C

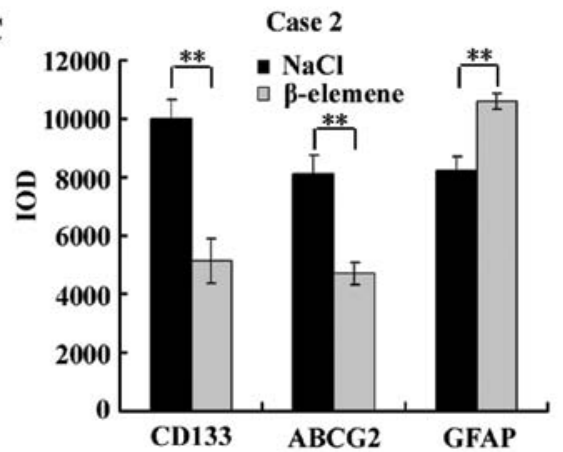

Figure 7. $\beta$-elemene decreases the expression of CD133 and ABCG2 and increases the expression of GFAP in vivo. (A) After the tumour tissues from GSCtransplanted nude mice treated with $\beta$-elemene or $\mathrm{NaCl}$ in the previous experiment were prepared following routine protocols, sample slides were probed with antibodies against CD133, ABCG2 and GFAP. (B and C) The IOD of each group was analysed using Image-Pro Plus 6.0 software (the same selected areas and magnifications in all the images) and illustrated by a vertical bar chart. The IODs for CD133 and ABCG2 were lower and the IOD for GFAP significantly higher in the $\beta$-elemene group than in the $\mathrm{NaCl}$ group. Serial sections stained with control antibodies were negative (data not shown). Values shown are the means $\pm \mathrm{SD}\left({ }^{*} \mathrm{P}<0.05,{ }^{* *} \mathrm{P}<0.01\right)$. Original magnifications $\mathrm{x} 200$.

areas and magnifications in all images), and then statistical analysis was performed. The IOD of each group is illustrated in a vertical bar chart (Fig. 7B and C). We found in this assay that the expression levels of CD133 and ABCG2 in the $\beta$-elemene group were significantly lower than in the $\mathrm{NaCl}$ group, and the expression level of GFAP in the $\beta$-elemene group was higher than in the $\mathrm{NaCl}$ group in both cases 1 and 2 . These results suggest that $\beta$-elemene decreased the expression of CD133 and ABCG2 but increased the expression of GFAP in vivo.

Treatment with $\beta$-elemene sensitizes GSCs to TMZ-induced cytotoxicity. As reported, GSCs were usually more resistant to TMZ cytotoxicity than normal glioblastoma cells (21). To determine whether treatment with $\beta$-elemene sensitizes GSCs to TMZ, GSC spheres were digested into single cells and then plated at a density of $5 \times 10^{3}$ cells/well in 96 -well plates. Cells were organized into the following three groups: control (untreated), TMZ (treated with $6 \mu \mathrm{g} / \mathrm{ml} \mathrm{TMZ}$ for $24 \mathrm{~h}$ ) and combination (treated with $6 \mu \mathrm{g} / \mathrm{ml} \mathrm{TMZ}$ and $100 \mu \mathrm{g} / \mathrm{ml} \beta$-elemene for $24 \mathrm{~h}$ ). After treatment with the drugs, CCK- 8 assays were performed to examine cell viability. Additionally, 18 nude mice were injected with GSCs to create tumours and divided into the following three groups (6 mice/group): $\mathrm{NaCl}$ (negative control, intraperitoneally injected with $50 \mathrm{mg} / \mathrm{kg} \mathrm{NaCl}$ for 1 week), TMZ (intraperitoneally injected with $33 \mathrm{mg} / \mathrm{kg}$ TMZ for 1 week) and combination (intraperitoneally injected with $33 \mathrm{mg} / \mathrm{kg}$ TMZ and $50 \mathrm{mg} / \mathrm{kg} \beta$-elemene for 1 week). The volumes of tumours were measured and calculated according to the formula: $\mathrm{V}=$ $1 / 2 \times$ largest diameter $\mathrm{x}$ smallest diameter ${ }^{2}$, every 3 days, and tumours were weighed on the 21st day. The results showed that 
A

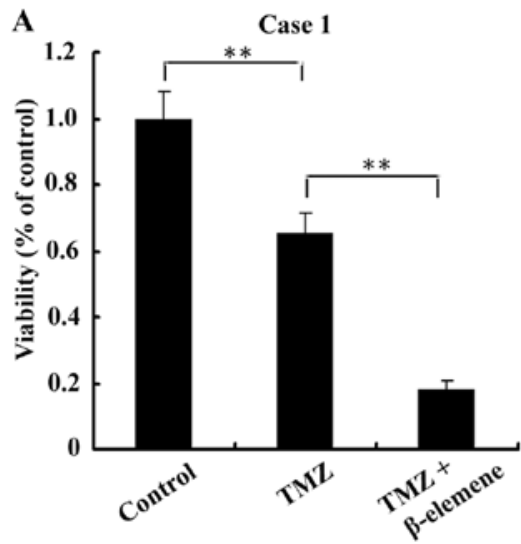

C

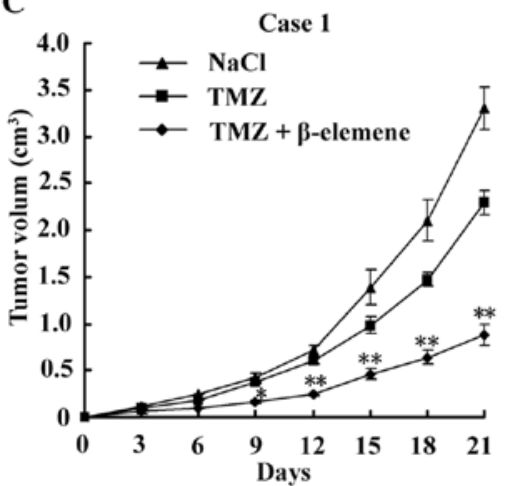

E

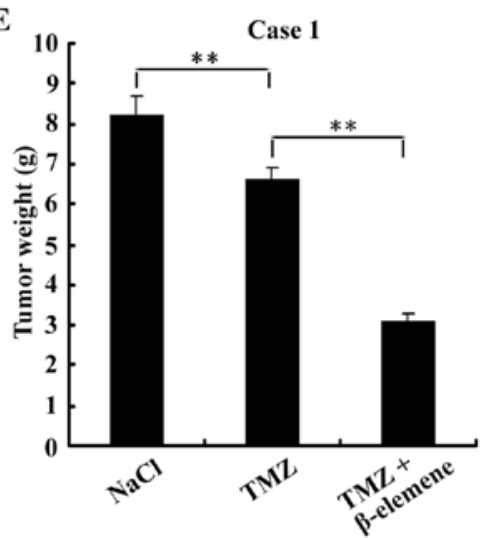

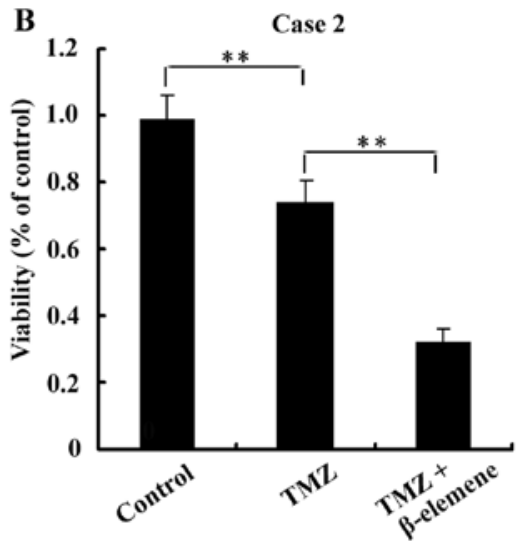

D

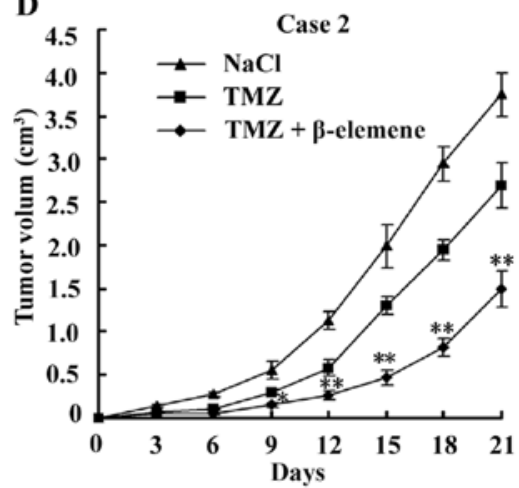

$\mathbf{F}$

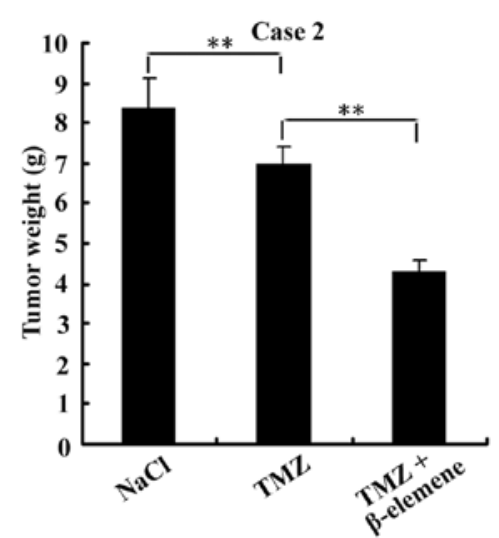

Figure 8. $\beta$-elemene increases the sensitivity of GSCs to TMZ-induced cytotoxicity in vivo and in vivo. (A and B) GSC spheres of case 1 and case 2 were digested into single cells and then plated in 96-well plates. CCK-8 assays were performed after treatment with the drugs. Cell viability was lower in the combination group than in either the control or TMZ groups. (C and D) The tumour volumes of nude mice were less in the combination group than in either the control or TMZ groups in case 1 and case 2. (E and F) The weights of tumours were less in the combination group than in either the control or TMZ groups in case 1 and case 2 . These findings suggest that treatment with $\beta$-elemene sensitized GSCs to TMZ-induced cytotoxicity in vitro and in vivo. Values shown are the means $\pm \mathrm{SD}\left({ }^{*} \mathrm{P}<0.05,{ }^{* *} \mathrm{P}<0.01\right)$.

cell viability was lower in the combination group than either the control or the TMZ groups (Fig. $8 \mathrm{~A}$ and $\mathrm{B}$ ). In the in vivo experiments, we found that both the volumes (Fig. 8C and D) and the weights (Fig. 8E and F) of tumours were less in the combination group than in either the control or TMZ groups. These findings suggest that $\beta$-elemene increased the sensitivity of GSCs to TMZ-induced cytotoxicity.

\section{Discussion}

It was reported that $\mathrm{CD} 133^{+}$cells tend to distribute around microvessels in glioblastoma tissues $(24,25)$. In this study, we found that $\mathrm{CD}_{133^{+}}$cells were not only assembled in some vascular walls (smaller cells) but also sparsely localized in other parts of glioblastoma tissues (larger cells). This difference in the cell distribution may be due to individual variability between the samples.

It has been suggested that GSCs are the source of malignant tumour development, recurrence and chemoresistance. Conventional chemotherapy will only kill the bulk of normal glioblastoma cells in a tumour but will leave the drug-resistant GSCs intact, resulting in tumour recurrence. A representative property of GSCs is the ability to form neurospheres in special NSCM. Thus, the effects of drugs on GSCs can be conve- 
niently evaluated by measuring cell proliferation, the ability to form neurospheres and the degree of neurosphere dispersion (26-29). We previously found that $\beta$-elemene can significantly reduce the size of tumours and prolong the life-span of patients without serious side effects and that it can inhibit glioblastoma growth through the activation of GMF $\beta$-MKK3/6-p38 and the downregulation of phosphorylated ERK1/2 in vitro and in vivo (17-21). In this study, we found that $\beta$-elemene inhibited the proliferation of GSCs in vitro and in vivo, decreased the formation of GSC spheres, and dispersed GSC spheres, which resulted in the fragmentation and death of some sphere cells. $\beta$-elemene also suppressed the ability of GSCs to form xenografts in nude mice. This is the first report of the effects of $\beta$-elemene on GSCs.

Human CD133 (prominin-1), a 120-kDa cell-surface protein, has been accepted as the standard marker for the identification of GSCs, although it may not be exclusive or the most ideal marker for this cell type (30-32). ATP-binding cassette subfamily G (ABCG) is a membrane pump that consumes ATP to excrete endogenous small molecules, such as cholesterol, ions and peptides, across cell membranes. ABCG also plays a pivotal part in detoxification and protection against xenobiotics by expelling drugs from the cell. NSCs and TSCs have been shown to express high levels of ABCG, which is normally inactive in more mature cells. It was shown that a major member of the ABCG family, ABCG2 (also known as BCRP1), is an important resistance-related molecule and GSC marker (8,33-36). The capacity for multipotent differentiation is a representative characteristic of GSCs. The expression of the astroglial cell marker GFAP was low in NSCs and GSCs and gradually increased during the cellular differentiation process. Therefore, the increase in the expression of GFAP generally indicated an enhancement of GSC differentiation. In this study, we found that the expression levels of CD133 and ABCG2 were significantly downregulated, whereas the expression of GFAP was increased by $\beta$-elemene in both GSC spheres in vitro and in nude mouse xenografts in vivo. These results suggested that $\beta$-elemene could impair the stemness of GSC spheres and promote their differentiation into normal glioblastoma cells.

TMZ is the most commonly used chemotherapeutic agent in glioblastoma treatment. It achieves its antitumour effect primarily by methylating the $\mathrm{O} 6$ position of guanine. TMZ can kill most glioblastoma cells and improves the overall survival and the progression-free survival of patients with glioblastoma undergoing surgical resection and radiotherapy (37-40). However, GSCs in glioblastoma tissues show incredible chemoresistance to TMZ treatment. It was reported that GSCs can survive even in the presence of $200 \mu \mathrm{M} \mathrm{TMZ}$ $(13,41,42)$. The maximum TMZ concentrations in the plasma of patients are between 27 and $50 \mu \mathrm{M}$ and are only $0.5-10 \mu \mathrm{M}$ in cerebrospinal fluid $(21,43,44)$, which suggests that it is virtually impossible to use TMZ to eliminate GSCs. Therefore, enhancing the sensitivity of GSCs to chemotherapeutic drugs is crucial for decreasing neoplasm recurrence and improving long-term prognosis. $\beta$-elemene is not only an antitumour agent but is also a chemosensitizer in the treatment against glioblastoma. $\beta$-elemene could partially reverse the multidrug resistance to adriamycin in SGC7901/Adr human gastric carcinoma cells (45). Similarly, the synergistic anticancer effects of $\beta$-elemene and cisplatin were observed in human laryngeal carcinoma-bearing nude mice and ovarian carcinoma cells $(46,47)$. We previously found that $\beta$-elemene can sensitize U87 human glioblastoma cells to cisplatin in vitro (19). In this study, we conducted experiments in vitro and in vivo and found that $\beta$-elemene sensitized GSC spheres to TMZ-induced cytotoxicity and enhanced the anti-proliferative effects of TMZ on nude mouse xenografts.

However, the chemosensitization mechanism of $\beta$-elemene remains unclear. We speculate that the following aspects may be involved. First, the differentiation of GSCs into normal glioblastoma cells induced by $\beta$-elemene improved the sensitivity of GSCs to antitumour drugs. Second, $\beta$-elemene downregulated the expression of the resistance-related protein ABCG2, which was likely to increase the intracellular accumulation of chemotherapeutics. Finally, the p38 mitogen-activated protein kinase (MAPK) and extracellular signal-regulated kinases 1 and 2 (ERK1/2)/BCL-2 signalling pathways are closely related to the drug resistance of various tumours (17-21). We previously reported that $\beta$-elemene could activate the GMF $\beta$-MKK3/6-p38 pathway and inhibit the ERK1/2/BCL-2 pathway, and this may also be a potential molecular mechanism underlying chemosensitization with $\beta$-elemene.

In conclusion, $\beta$-elemene impaired the stemness of GSC spheres, promoted their differentiation and sensitized GSCs to TMZ-induced cytotoxicity in vitro and in vivo. $\beta$-elemene will hopefully become a valuable agent to enhance the effects of radiotherapy and chemotherapy and improve the long-term prognosis of patients with glioblastoma.

\section{Acknowledgments}

This research was supported by the Post-doctoral Science Foundation of China (No. 2012M521921) and the Liaoning Province Science and Technology Key Projects (No. 2013225089). We also thank all the colleagues in our research group for their generous support.

\section{References}

1. Wen PY and Kesari S: Malignant gliomas in adults. N Engl J Med 359: 492-507, 2008.

2. Van Meir EG, Hadjipanayis CG, Norden AD, Shu HK, Wen PY and Olson JJ: Exciting new advances in neuro-oncology: the avenue to a cure for malignant glioma. CA Cancer J Clin 60: 166-193, 2010.

3. Omuro A and DeAngelis LM: Glioblastoma and other malignant gliomas: a clinical review. JAMA 310: 1842-1850, 2013.

4. Shah U and Morrison T: A review of the symptomatic management of malignant gliomas in adults. J Natl Compr Cancer Netw 11: 424-429, 2013.

5. Eyüpoglu IY, Buchfelder M and Savaskan NE: Surgical resection of malignant gliomas-role in optimizing patient outcome. Nat Rev Neurol 9: 141-151, 2013.

6. Grimm SA and Chamberlain MC: Brainstem glioma: a review. Curr Neurol Neurosci Rep 13: 346, 2013.

7. He J, Liu Y and Lubman DM: Targeting glioblastoma stem cells: cell surface markers. Curr Med Chem 19: 6050-6055, 2012.

8. Ding XW, Wu JH and Jiang CP: ABCG2: a potential marker of stem cells and novel target in stem cell and cancer therapy. Life Sci 86: 631-637, 2010.

9. Singh SK, Hawkins C, Clarke ID, et al: Identification of human brain tumour initiating cells. Nature 432: 396-401, 2004.

10. Yuan X, Curtin J, Xiong Y, et al: Isolation of cancer stem cells from adult glioblastoma multiforme. Oncogene 23: 9392-9400, 2004. 
11. Bidlingmaier S, Zhu X and Liu B: The utility and limitations of glycosylated human CD133 epitopes in defining cancer stem cells. J Mol Med (Berl) 86: 1025-1032, 2008.

12. Beier D, Schulz JB and Beier CP: Chemoresistance of glioblastoma cancer stem cells - much more complex than expected. Mol Cancer 10: 128, 2011.

13. Liu G, Yuan X, Zeng Z, et al: Analysis of gene expression and chemoresistance of $\mathrm{CD} 133^{+}$cancer stem cells in glioblastoma. Mol Cancer 5: 67, 2006.

14. Chen SL, You J and Wang GJ: Supercritical fluid extraction of $\beta$-elemene under lower pressure. Se Pu 19: 179-181, 2001 (In Chinese).

15. Zhang X, Zhang Y and Li Y: $\beta$-elemene decreases cell invasion by upregulating E-cadherin expression in MCF-7 human breast cancer cells. Oncol Rep 30: 745-750, 2013.

16. Bao F, Qiu J and Zhang H: Potential role of $\beta$-elemene on histone $\mathrm{H} 1$ in the $\mathrm{H} 22$ ascites hepatoma cell line. Mol Med Rep 6: 185-190, 2012.

17. Zhu T, Zhao Y, Zhang J, Li L, Zou L, Yao Y and Xu Y: $\beta$-Elemene inhibits proliferation of human glioblastoma cells and causes cell-cycle G0/G1 arrest via mutually compensatory activation of MKK3 and MKK6. Int J Oncol 38: 419-426, 2011.

18. Yao YQ, Ding X, Jia YC, Huang CX, Wang YZ and Xu YH: Anti-tumor effect of beta-elemene in glioblastoma cells depends on p38 MAPK activation. Cancer Lett 264: 127-134, 2008.

19. Zhu T, Xu Y, Dong B, Zhang J, Wei Z, Xu Y and Yao Y: $\beta$-elemene inhibits proliferation of human glioblastoma cells through the activation of gliamaturation factor $\beta$ and induces sensitization to cisplatin. Oncol Rep 26: 405-413, 2011

20. Yao YQ, Xu YH, Lu J, Zhou HY and Wang YZ: Effect of p38 MAPK on elemene-induced cell cycle arrest in C6 glioblastoma cells. Zhonghua Yi Xue Za Zhi 88: 56-58, 2008 (In Chinese).

21. Zhao YS, Zhu TZ, YQ Yao, et al: $\beta$-elemene inhibits Hsp90/ Raf-1 molecular complex inducing apoptosis of glioblastoma cells. J Neurooncol 107: 307-314, 2012.

22. Zhang L and Zhang RH: Investigation of Tca8113 cell lines differentiation induced by $\beta$-elemene. J Oral Sci Res 18: 307-309, 2002 (In Chinese)

23. Fang Z, Chen HS, Chen HB and Liu YM: $\beta$-Elemene induced differentiation of PLA801D cell lines. Chin J Gerontol 28 768-769, 2008 (In Chinese).

24. Wang HM, Yang XJ, Dong XT, et al: Correlation between the distribution of CD133-positive cells and the proliferation of microvessels in glioblastoma multiforme. Zhonghua Yi Xue Za Zhi 91: 781-785, 2011 (In Chinese).

25. He H, Niu CS and Li MW: Correlation between glioblastoma stem-like cells and tumor vascularization. Oncol Rep 27: 45-50, 2012.

26. Carrasco-Garcia E, Sampron N, Aldaz P, et al: Therapeutic strategies targeting glioblastoma stem cells. Recent Pat Anticance Drug Discov 8: 216-227, 2013.

27. Gal H, Makovitzki A, Amariglio N, Rechavi G, Ram Z and Givol D: A rapid assay for drug sensitivity of glioblastoma stem cells. Biochem Biophys Res Commun 358: 908-913, 2007.

28. Sampetrean O and Saya H: Characteristics of glioma stem cells. Brain Tumor Pathol 30: 209-214, 2013.

29. Salmaggi A, Boiardi A, Gelati M, et al: Glioblastoma-derived tumorospheres identify a population of tumor stem-like cells with angiogenic potential and enhanced multidrug resistance phenotype. Glia 54: 850-860, 2006.
30. Choy W, Nagasawa DT, Trang A, Thill K, Spasic M and Yang I: CD133 as a marker for regulation and potential for targeted therapies in glioblastoma multiforme. Neurosurg Clin N Am 23: 391-405, 2012

31. Zhang B, Sun J, Yu SP, et al: $\mathrm{Racl}^{+}$cells distributed in accordance with $\mathrm{CD} 133^{+}$cells in glioblastomas and the elevated invasiveness of $\mathrm{CD}_{133^{+}}$glioma cells with higher Rac1 activity. Chin Med J (Engl) 125: 4344-4348, 2012.

32. Singh SK, Clarke ID, Terasaki M, Bonn VE, Hawkins C, Squire J and Dirks PB: Identification of a cancer stem cell in human brain tumors. Cancer Res 63: 5821-5828, 2003.

33. Dean M, Fojo T and Bates S: Tumour stem cells and drug resistance. Nat Rev Cancer 5: 275-284, 2005.

34. Bleau AM, Huse JT and Holland EC: The ABCG2 resistance network of glioblastoma. Cell Cycle 8: 2936-2944, 2009.

35. Scharenberg CW, Harkey MA and Torok-Storb B: The ABCG2 transporter is an efficient Hoechst 33342 efflux pump and is preferentially expressed by immature human hematopoietic progenitors. Blood 99: 507-512, 2002.

36. Nakanishi $T$ and Ross DD: Breast cancer resistance protein (BCRP/ABCG2): its role in multidrug resistance and regulation of its gene expression. Chin J Cancer 31: 73-99, 2012.

37. Stupp R, Mason WP, van den Bent MJ, et al: Radiotherapy plus concomitant and adjuvant temozolomide for glioblastoma. $\mathrm{N}$ Engl J Med 352: 987-996, 2005.

38. Zhang L, Wu X, Xu T, Luo C, Qian J and Lu Y: Chemotherapy plus radiotherapy versus radiotherapy alone in patients with anaplastic glioma: a systematic review and meta-analysis. J Cancer Res Clin Oncol 139: 719-726, 2013.

39. Hau P, Koch D, Hundsberger T, et al: Safety and feasibility of long-term temozolomide treatment in patients with high-grade glioma. Neurology 68: 688-690, 2007.

40. Hart MG, Garside R, Rogers G, Stein K and Grant R: Temozolomide for high grade glioma. Cochrane Database Syst Rev 4: CD007415, 2013

41. Eramo A, Ricci-Vitiani L,Zeuner A, et al: Chemotherapy resistance of glioblastoma stem cells. Cell Death Differ 13: 1238-1241, 2006.

42. Clement V, Sanchez P, de Tribolet N, Radovanovic I and Ruiz Altaba A: HEDGEHOG-GLI1 signaling regulates human glioma growth, cancer stem cell self-renewal, and tumorigenicity. Curr Biol 17: 165-172, 2007.

43. Brada M, Judson I, Beale P, et al: Phase I dose-escalation and pharmacokinetic study of temozolomide ( $\mathrm{SCH}$ 52365) for refractory or relapsing malignancies. Br J Cancer 81: 1022-1030, 1999.

44. Ostermann S, Csajka C, Buclin T, Leyvraz S, Lejeune F, Decosterd LA and Stupp R: Plasma and cerebrospinal fluid population pharmacokinetics of temozolomide in malignant glioma patients. Clin Cancer Res 10: 3728-3736, 2004

45. Zhang H, Song N, Liu YP, Qu XJ, Hou KZ, Teng YE and Zhang JD: Reversal of resistance to adriamycin in human gastric cancer by $\beta$-elemene. J Chian Med Univ 40: 968-993, 2011 (In Chinese).

46. Li X, Wang G, Zhao J, et al: Antiproliferative effect of betaelemene in chemoresistant ovarian carcinoma cells is mediated through arrest of the cell cycle at the G2-M phase. Cell Mol Life Sci 62: 894-904, 2005 .

47. Tao L, Zhou L, Zheng LY and Yao M: Inhibition of eIF families expression and angiogenesis for human laryngeal carcinoma by elemene administration. Zhonghua Er Bi Yan Hou Tou Jing Wai Ke Za Zhi 40: 840-845, 2005 (In Chinese). 